\title{
Special Issue on Higher-Level Cognition and Computation
}

\author{
Marco Ragni $^{1} \cdot$ Frieder Stolzenburg $^{2}$
}

Published online: 7 July 2015

(c) Springer-Verlag Berlin Heidelberg 2015

One distinctive feature between humans and other animals, recent AI or robotic systems is higher-level cognition. This field connects many research fields and touches philosophy, computer science, artificial intelligence, psychology and neuroscience among others. This special issue highlights current research directions from an AI perspective. In a combination of survey articles, interviews with experts from AI, technical and project reports, this still emerging field becomes more and more precise. In each and every article of this special issue, connections to cognition and psychological aspects are drawn and it becomes obvious that many authors do not approach the problems from a single perspective, but all of them approach the problems from a multi- and even transdisciplinaric perspective. Most contributions deal with approaches from knowledge and reasoning and try to draw connections from AI to cognitive science. Fundamental questions about the way of how to measure machine intelligence, probabilistic methods for higher-level cognition, inductive reasoning, and the evaluation of spatial reasoning are addressed. These methods can approximate, imitate, and simulate human reasoning.

Frieder Stolzenburg

fstolzenburg@hs-harz.de

Marco Ragni

ragni@cs.uni-freiburg.de

1 Research Group on the Foundations of Artificial Intelligence, Institute for Informatik, Albert-Ludwigs-Universität Freiburg, Georges-Köhler-Allee 52, 79110 Freiburg, Germany

2 Automation and Computer Sciences Department, Harz University of Applied Sciences, Friedrichstr. 57-59, 38855 Wernigerode, Germany
The technical contributions cover a survey of higherlevel cognition and computation (Marco Ragni, Frieder Stolzenburg) and research about an unbiased extension of probabilistic knowledge in the medical domain, applying the so-called principle of maximum entropy (Christoph Beierle, Gabriele Kern-Isberner, Marc Finthammer, Nico Potyka). In a further technical contribution, Mathias Winther Madsen investigates limits and potentials of a multiagent probability theory and its implication for statistical consistency problems. Two research projects, namely the RatioLog project investigating rational extensions of logical reasoning (Ulrich Furbach, Claudia Schon, Frieder Stolzenburg, Karl-Heinz-Weis, Claus-Peter Wirth) and qualitative and semi-quantitative inductive reasoning with conditionals (Christian Eichhorn, Gabriele Kern-Isberner), are presented. In the former project, an application of higher-level cognition is considered, namely deep question answering, where techniques from information retrieval and automated reasoning are combined in a system that aims at implementing rational reasoning. The latter project investigates aspects of common-sense reasoning especially for inductive reasoning and analyzes limits and potentials of qualitative and semi-quantitative semantics covering preferential models and so-called ranking functions.

Human intelligence has often been the Mètre des Archives for artificial intelligence, but the application of psychometric tests to measure machine intelligence has been disputed. Tarek Besold, José Hernández-Orallo, and Ute Schmid discuss this question. Finally, two interviews with Bernhard Nebel and Wolfram Burgard shed light on applications, new frontiers, and expectations from an AI perspective at research in higher-level cognition and computation.

Spatial reasoning has often been seen as the drosophila for human reasoning in general. Therefore, a recent book 
by Markus Knauff about this topic with the title Space to Reason: A Spatial Theory of Human Thought is reviewed here by Yacin Hamami.

All contributions have the clear motivations to unravel some of the mysteries of the human mind and to apply techniques of higher-level cognition, also addressing computational issues, e.g. from logical or probabilistic reasoning. Still it becomes obvious that the journey into an undiscovered country has just begun.

\section{Content}

\subsection{Technical Contributions}

- Higher-level cognition and computation: A survey (Marco Ragni, Frieder Stolzenburg).

- Extending and completing probabilistic knowledge and beliefs without bias (Christoph Beierle, Gabriele KernIsberner, Marc Finthammer, Nico Potyka).

- On the consistency of approximate multi-agent probability theory (Mathias Winther Madsen).

\subsection{Research Projects}

- The RatioLog project: Rational extensions of logical reasoning (Ulrich Furbach, Claudia Schon, Frieder Stolzenburg, Karl-Heinz-Weis, Claus-Peter Wirth).

- Qualitative and semi-quantitative inductive reasoning with conditionals (Christian Eichhorn, Gabriele KernIsberner).

\subsection{Discussion}

- Can machine intelligence be measured in the same way as human intelligence? (Tarek Besold, José HernándezOrallo, Ute Schmid).

\subsection{Interviews}

- Interview with Wolfram Burgard: The pleasure will be always on our side (Marco Ragni and Christian BeckerAsano).

- Interview with Bernhard Nebel: To make the world a better place (Marco Ragni).

\subsection{Book Review}

- Markus Knauff: Space to reason-a spatial theory of human thought (Yacin Hamami).

\section{Service}

\subsection{Conferences}

- CogSci 2015-mind, technology, and society http://cognitivesciencesociety.org/conference2015/.

- EAP CogSci 2015-EuroAsianPacific joint conference on cognitive science http://www.eapcogsci2015.it/.

- IJCAI 2016-International joint conference on artificial intelligence http://ijcai-16.org/.

- KogWis 2015-Biannual meeting of the German Cognitive Science Society http://www.gk-ev.de/.

- KI 2015-Künstliche Intelligenz http://ki2015.computational-logic.org/.

\subsection{Workshops}

- Bridging 2015-Workshop on bridging the gap between human and automated reasoning http://ratiolog.uni-koblenz.de/bridging.html.

- DKB 2015-Workshop on dynamics of knowledge and belief

KIK 2015-Workshop KI \& Kognition http://www.fernuni-hagen.de/wbs/dkbkik2015.html.

- MIWAI 2015-Multi-disciplinary international workshop on artificial intelligence http://khamreang.msu.ac.th/miwai15/.

\subsection{Organizations}

- CogSci-Cognitive Science Society http://cognitivesciencesociety.org/.

- EUCOG-European network for the advancement of artificial cognitive systems, interaction and robotics http://www.eucognition.org/.

- GI-FG Kognition-GI-Fachgruppe Kognition am Fachbereich Künstliche Intelligenz

http://imodspace.iig.uni-freiburg.de/FachgruppeKogni tion/.

- GK-Gesellschaft für Kognitionswissenschaft http://www.gk-ev.de/.

\subsection{Journals}

- Artificial intelligence.

- Cognition.

- Cognitive computation journal.

- Cognitive processing. 
- Cognitive science.

- Cognitive systems research.

- Journal of experimental and theoretical artificial intelligence.

- Spatial cognition and computation: An interdisciplinary journal.

\subsection{Books}

- Anderson, J.R.: The architecture of cognition. Harvard University Press, Cambridge, MA (1983).

- Anderson, J.R.: How can the human mind occur in the physical universe? Oxford University Press, New York (2007).

- Ariely, D.: Predictably irrational: The hidden forces that shape our decisions, revised and updated edition. Harper Collins (2010).
- Knauff, M.: Space to reason: A spatial theory of human thought. MIT Press (2013).

- Laird, J.E.: The Soar cognitive architecture. MIT Press (2012).

- Mueller, E.T.: Commonsense reasoning, 2nd edn. Morgan Kaufmann, San Francisco (2014).

\subsection{Summer Schools}

- BCBT2015-Barcelona cognition, brain and technology summer school http://bcbt.upf.edu/bcbt15/.

- IK-Interdisciplinary college Günne

- ICCL Summer school 2015 'reasoning', Dresden http://ddll.inf.tu-dresden.de/web/SummerSchool2015/ en. 